\title{
Intertester reliability of brachial artery flow-mediated vasodilation using upper and lower arm occlusion in healthy subjects
}

\author{
Ludmila M Cosio-Lima' \\ Richard Seip ${ }^{2}$ \\ Paul D Thompson² \\ Marie A Lagasse ${ }^{2}$ \\ Tabitha H Hodges' \\ 'University of West Florida, Pensacola, \\ FL, USA; ${ }^{2}$ Hartford Hospital, Hartford, \\ CT, USA
}

Correspondence: Ludmila M Cosio-Lima University of West Florida, I 1000

University Parkway, Bldg. \#72 room 220,

Pensacola, FL 325 I 4, USA

Tel + I 8504737350

Email Icosiolima@uwf.edu

\begin{abstract}
The assessment of endothelial function as brachial artery flow-mediated vasodilatation is a widely used technique that determines the effect of risk factor intervention and may have the potential to predict the clinical benefit of antiatherogenic therapy. Previous studies suggest that flow-mediated dilation is greater using the upper-arm occlusion technique, but no data are available to compare intertester reliability between technicians. This study was undertaken to compare the amount of hyperemia between upper and lower occlusion techniques and to determine reproducibility between testers. Nineteen healthy adults, ages 25 to 50 , were included in the study. Brachial artery vasodilatation was measured 1 and 3 minutes post cuff deflation and was compared with the baseline and expressed as a percent change. There was a tester effect in the percent change in diameter across all measurements. The results of this study reveal inconsistencies between testers when using a blood pressure cuff to induce hyperemia for the assessment of endothelial function through brachial artery flow-mediated vasodilation. However, upper arm as compared to lower arm blood pressure cuff occlusion results in significantly greater hyperemia and vasodilatation, even though there was a difference in measurements between testers.
\end{abstract}

Keywords: endothelial function, flow-mediated vasodilatation, hyperemia

Over the past decade, a noninvasive technique has evolved to evaluate flow-mediated vasodilatation (FMD), an endothelium-dependent function, in the brachial artery (Anderson and Mark 1989; Laurent et al 1990; Celarmajer et al 1992; Sorensen et al 1995). This noninvasive procedure utilizes high resolution ultrasound imaging in which brachial arteries are scanned under baseline conditions and during hyperemia (Bots et al 2005). Induction of hyperemia occurs through the inflation and deflation of a sphygmomanometer (blood pressure) cuff, with the occlusion located either at the lower or upper arm and generally lasts 5 minutes before deflation (Bots et al 2005). Endothelial function, which through flow-mediated vasodilatation is expressed as a percentage change in diameter of vessels from baseline conditions to maximum diameter during hyperemia, is abnormal in the presence of coronary risk factors and coronary artery disease, and might be an important initiating factor in the pathogenesis of arteriosclerosis (Glasser et al 1996; Celermajer 1997; Vogel et al 2000; Bots et al 2005). The assessment of endothelial function as brachial artery flow-mediated vasodilatation is also important to determine the effect of risk factor intervention and may have the potential to predict the clinical benefits of antiatherogenic therapy. Bots and colleagues (2005) conducted a literature review in which they "evaluated technical and operative factors and their relationship to the variety in absolute values of flow-mediated vasodilatation across studies published between 1992 and 2001" (Bots et al 2005, p 364). They concluded that the technical aspects, which they described as 
location and duration of the occlusion, potentially explain the variability between and within previous studies utilizing this technique (Bots et al 2005). Additionally, two reports suggested that the percent change in flow-mediated vasodilatation is greater using the upper arm occlusion technique versus the lower arm occlusion (Mannion et al 1998; Vogel et al 2000). While previous studies suggest variability in the technical aspects, no data are available to compare intertester reliability between technicians. Therefore, we studied the reproducibility of brachial artery reactivity in different subjects under several conditions. Both upper and lower arm cuff inflation methods were examined by two different certified testers with equal experience. This study was undertaken to compare the amount of hyperemia between upper and lower occlusion techniques and to determine reproducibility of measurements between testers.

We studied 19 healthy adults ( 7 men and 12 women aged 25 to 50 years) without risk factors other than age and gender (eg, males over the age of 45). These subjects had serum cholesterol $<200 \mathrm{mg} / \mathrm{dl}$, blood pressure $<132 / 90 \mathrm{mmHg}$ (off medications), no history of cigarette smoking or diabetes mellitus, and none were taking vasoactive medications at the time of this study.

Each technician performed two upper and two lower occlusions per subject on separate occasions, and all scans were completed within four weeks. Flow-mediated vasodilatation was assessed between 8:00 and 10:00 A.M. at the Endothelial Function Laboratory at Hartford Hospital. Subjects were asked to refrain from consuming stimulants such as caffeine, which might have an effect on endothelial function, for $24 \mathrm{hr}$ before the testing session. Subjects were also asked to fast overnight (approximately 12 hours) before each scan and refrain from using Sildenafil (Viagra, which might have a vasoactive effect and can react with the nitroglycerin pills used during the brachial artery reactivity assessment). All subjects included in the study provided informed consent as approved by the Institutional Review Board of Hartford Hospital and were measured a total of four times by two different technicians.

Endothelial function was determined using highresolution ultrasound of the brachial artery and ischemic forearm occlusion. For this procedure, subjects rested for 10 minutes in the supine position. ECG leads were attached to monitor heart rate and for image acquisition. Images were acquired in correspondence of the R wave of the ECG. A baseline blood pressure was taken in the left arm. The right brachial artery was imaged for a baseline measurement two inches above the antecubital fossa using an Acuson Aspen ultrasonography machine (Acuson Corp, Mountain View, CA, USA). A Hokanson Rapid Cuff Inflator (DE Hokanson Inc, Bellevue, WA, USA) was placed over the right forearm, below the elbow for lower occlusion and below the axillary for upper occlusion. The cuff was inflated $60 \mathrm{mmHg}$ above the resting systolic pressure for 5 minutes. The occluding pressure was released and the artery imaged 1 and 3 minutes post deflation. Following the endothelial dependent assessment, the subject rested for 15 minutes to allow the effects of cuff occlusion to reside. A second baseline measurement was taken following the 15-minute rest period. A physician or nurse administered $0.4 \mathrm{mg}$ of sublingual nitroglycerin to the subject. Nitroglycerin was used to show independent vasodilatation on the smooth muscle of the artery. Vessel diameter was recorded 3 and 5 minutes following nitroglycerin administration. Blood pressure of the subject was monitored in the supine, seated, and standing positions following the ultrasound for possible hypotensive response. Arterial crosssectional diameter was measured from the near endothelialluminal surface to the distal luminal surface. Endothelial function was quantified as the percent change in brachial artery diameter (vasodilatation) by dividing the change in diameter by the baseline value after 1, 3, and 5 minutes of lower and upper arm ischemic occlusion.

Descriptive statistics were calculated for anthropometrics and dependent variables (Table 1). A 2 x 2 repeated ANOVAs was used to analyze the data. Technician, (Tester 1 versus Tester 2), an independent groups factor, and position (upper and lower), a repeated measures factor, were the independent variables. The dependent variables were the percent change in dependent and independent endothelial dilatation. Sidak post hoc comparisons were conducted following significant interactions. A level of $\mathrm{p}<0.05$ was considered statistically significant.

The results indicated the effects of position and technician on reactivity. As expected, upper arm occlusion resulted in a significantly greater $(\mathrm{p}<0.05)$ percent change in dilation at 1 minute and 3 minutes post occlusion when compared to lower-arm occlusion, regardless of the tester (9.52 and

Table I Age and anthropometric measurements in the subject group $(\mathrm{N}=19)($ mean $\pm \mathrm{SD})$

\begin{tabular}{ll}
\hline Variables & Mean \pm SD \\
\hline Age (years) & $36.75 \pm 7.92$ \\
Height $(\mathrm{m})$ & $1.70 \pm 11.72$ \\
Weight $(\mathrm{kg})$ & $73.27 \pm 21.83$ \\
BMI $\left(\mathrm{kg} / \mathrm{m}^{2}\right)$ & $25.33 \pm 7.44$ \\
\hline
\end{tabular}

Abbreviations: BMI, body mass Index; SD, standard deviation. 
9.32 versus 6.7 and $4.32 \%$ at 1 minute post occlusion; 7.5 and 8.3 versus 4.9 and $1.9 \%$ at 3 minutes post occlusion). Cuff placement did not affect the response to NTG ( $p>0.05)$.

For both testers, upper arm cuff placement resulted in a greater percent change at 1 minute and 3 minutes when compared with lower arm cuff placement. There was a tester effect in percent change diameter across all measurements (post occlusion at 1 minute and 3 minutes; post NTG at 3 minutes and 5 minutes) (Tables 2 and 3 ). Tester 2 recorded significantly $(\mathrm{p}<0.05)$ higher percent changes in diameter in the upper occlusion than Tester 1 (6.7 versus 4.3\%).

Since the inception of the description of brachial artery flow-mediated vasodilatation via ultrasound by Anderson and Mark (1989), several studies have utilized the technique for a variety of purposes. Some studies have reported greater vasodilatation employing upper arm versus lower arm occlusion in the same subjects (Sorensen et al 1995; Mannion et al 1998). Although upper arm cuff occlusion was initially used as the hyperemic stimulus, several laboratories and technicians changed to lower arm occlusion, because it caused less patient discomfort, and it was easier to visualize the brachial artery during and immediately after cuff deflation (Mannion et al 1998). In addition, concerns were raised regarding whether the upper arm approach produced "ischemic" vasodilatation in addition to flow-mediated vasodilatation.

This study found that 5 minutes of upper arm cuff occlusion produced significantly more brachial artery hyperemia than lower arm cuff occlusion. Additionally, there was a difference between testers for the upper arm occlusion, as one tester recorded higher percent changes in the upper arm occlusion position. The difference found in upper arm occlusion values in this study between testers could be due the more challenging upper arm occlusion technique. It has been demonstrated that accurate data acquisition of the upper arm is technically more challenging since the image

Table 2 Comparison of upper versus lower arm occlusion between testers (dependent dilatation)

\begin{tabular}{lll}
\hline & Tester I & Tester 2 \\
\hline $\begin{array}{lll}\text { Baseline absolute diameter (mm) } \\
\text { Upper occlusion }\end{array}$ & $3.67 \pm 0.55$ & $3.5 \pm 0.70$ \\
$\begin{array}{l}\text { Lower occlusion } \\
\text { Percent change diameter (\%) }\end{array}$ & $3.83 \pm 0.88$ & $3.6 \pm 0.68$ \\
Upper I-min post occlusion (\%) & $9.52 \pm 4.25^{*}$ & $9.36 \pm 6.15^{*}$ \\
Lower I-min post occlusion (\%) & $6.68 \pm 4.03$ & $4.32 \pm 3.38$ \\
Upper 3-min post occlusion (\%) & $7.51 \pm 4.47^{*}$ & $8.33 \pm 7.90^{*}$ \\
Lower 3-min post occlusion (\%) & $4.86 \pm 4.06$ & $1.87 \pm 2.65$ \\
\hline
\end{tabular}

Notes: Values are means \pm SD. ${ }^{*} \mathrm{p}<0.05$ vs. baseline.
Table 3 Comparison of upper versus lower arm occlusion between testers (independent dilatation)

\begin{tabular}{lll}
\hline & Tester I & Tester 2 \\
\hline Baseline 2 absolute diameter (mm) & & \\
Upper occlusion & $3.71 \pm 0.62$ & $3.73 \pm 0.86$ \\
Lower occlusion & $4.07 \pm 1.1 \mathrm{I}$ & $3.67 \pm 0.83$ \\
Percent change diameter (\%) & & \\
NTG upper 3-min post occlusion (\%) & $18.40 \pm 5.88^{*}$ & $17.78 \pm 8.13^{*}$ \\
NTG lower 3-min post occlusion (\%) & $14.90 \pm 5.24$ & $16.80 \pm 7.14$ \\
NTG upper 5-min post occlusion (\%) & $21.67 \pm 5.42^{*}$ & $17.26 \pm 8.0 I^{*}$ \\
NTG lower 5-min post occlusion (\%) & $17.97 \pm 5.62$ & $19.69 \pm 6.18$ \\
\hline
\end{tabular}

Abbreviation: NTG, nitroglycerin.

Notes: Values are means \pm SD. ${ }^{*} \mathrm{p}<0.05$ vs. baseline.

is distorted by a collapse of the brachial artery and a shift of tissue (Corretti et al 1995).

In the present study, both testers were trained in the principles and technical aspects of 2D and Doppler ultrasonography, but it has been observed that there is a learning curve that requires several months and depends on the technical skills of the individual and the frequency with which the technique is performed (Corretti et al 1995, 2002). Meticulous attention to detail is necessary since small shifts in the angle of approach in the ultrasound beam may alter the diameter measurements substantially.

It is entirely plausible that a change in the hyperemic stimulus could also contribute to differences observed between testers. When the cuff is placed on the upper part of the arm, reactive hyperemia typically elicits a greater percent change in diameter compared with that of lower arm cuffplacement. The greater hyperemia observed in the upper-arm occlusion might be due to a greater flow stimulus resulting from recruitment of more resistance vessels. The flow stimulus should be consistent since any change in dilatation of the conduit artery may be related to changes in flow rather than improvement in vessel dilatation. A minimal difference in cuff placement for the upper occlusion between testers in the present study might have accounted for the different measurements observed.

Our study demonstrates that upper arm compared to lower arm blood pressure cuff occlusion when undertaken to induce hyperemia for the assessment of brachial artery flow-mediated vasodilatation results in significantly greater hyperemia. While other studies have revealed similar results, our study is the first to compare intertester reliability of assessment in both cuff positions. The variability and inconsistencies in absolute values between previous studies have been displayed as due to technical aspects, such as the location and duration of the occlusion (Bots et al 2005). In our 
study, the greater hyperemia using upper arm cuff placement is shown as independent of testers. However, there were differences in post occlusion measurements between testers, and this suggests that regardless of techniques used, interpretation of ultrasound measurements require adequate training and technique skills. Although higher dilatation responses with the upper arm occlusion occur regardless of the tester, we recommend that intertester variability in image acquisition and analysis be periodically reassessed, as intertester variability might be a causative factor in the great variation between absolute values of flow-mediated vasodilatation across studies. We studied a healthy population, but as endothelial function is central in determining prognosis and treatment interventions in several special populations (eg, coronary heart disease and diabetes mellitus), it is of paramount importance that all technicians acquire the skills and training necessary to obtain accurate assessments. Future research needs to be conducted to evaluate intertester reliability in flow-mediated vasodilatation in upper and lower arm occlusion and might consider using a special population.

\section{References}

Bots ML, Westerink J, Rabelink TJ, et al. 2005. Assessment of flow-mediated vasodilation (FMD) of the brachial artery: effects of technical aspects of the FMD measurement on the FMD response. Eur Heart J, 26:363-8.

Celermajer DS. 1997. Endothelial function: Does it matter? It is reversible? J AM Coll Cardiol, 30:325.

Celarmajer DS, Sorensen KE, Georgakopoulos C, et al. 1992. Non-invasive detection of endothelial dysfunction in children and adults at risk of atherosclerosis. Lancet, 340:1111-15.

Corretti MC, Anderson TJ, Benjamin EJ, et al. 2002. Guidelines for the ultrasound assessment of endothelial-dependent flow-mediated vasodilation of the brachial artery. J Amer Col Cardiol, 39:257-65.

Corretti MC, Plotnick GD, Vogel RA. 1995. Technical aspects of evaluation brachial artery vasodilatation using high-frequency ultrasound. Amer Physiol Soc, 268:1397-H1404.

Glasser SP, Selwyn AP, Ganz P. 1996. Atherosclerosis: Risk factors and the vascular endothelium. Am Heart J, 131:379-84.

Laurent S, Lacolley P, Brunel P, et al. 1990. Flow-dependent vasodilatation of brachial artery in essential hypertension. Am J Physiol Heart Circ Physiol, 258: H1004-H1011.

Mannion TC, Vita JA, Keaney JF, et al. 1998. Non-invasive assessment of brachial artery endothelial vasomotor function: the effect of cuff position on level of discomfort and vasomotor responses. Vasc Med, 3:263-7.

Sorensen KE, Celermajer DS, Spiegelhalter DJ, et al. 1995. Non-invasive measurement of human endothelium dependent arterial responses: accuracy and reproducibility. Br Heart $J, 74: 247-53$.

Vogel RA, Corretti MC, Plotnick GD. 2000. A comparison of brachial artery flow-mediated vasodilatation using upper and lower arm occlusion in subjects with and without coronary risk factors. Clin Cardiol, 23:571-2. 